\title{
CALEB WiLliams, o L’UMANITÀ DIVISA: LETTERATURA E POLITICA IN WILLIAM GODWIN
}

\section{Abstract}

Storico dai molteplici interessi, bibliotecario, musicista, uomo di cultura e grande modernista, studioso, in particolare, dell'Italia settecentesca, Calogero Farinella ci ha prematuramente lasciati nel giugno del 2019, a poco più di sessanta anni. Le sue pubblicazioni sulla Genova del Settecento e sulla scienza illuminista (veronese specialmente: Francesco Bianchini, Anton Mario Lorgna e l'Accademia dei Quaranta) fanno e faranno sempre data negli studi di storia della cultura. Allievo tra i più brillanti di Salvatore Rotta (1926-2001), dal quale mutuò la passione per il XVIII secolo in particolare, Farinella si laureò sotto la sua guida su William Godwin (1756-1836), padre di Mary Shelley, filosofo e scrittore politico di area libertaria, che, con la sua fondamentale opera, marchia a fuoco il passaggio, in Gran Bretagna, dai Lumi al Romanticismo. Sulla figura e gli scritti di Godwin, ricavandoli dalla propria dissertazione di laurea, Farinella estrasse e pubblicò, negli anni Ottanta del secolo scorso, due articoli, apparsi nel volume della «Miscellanea storica ligure» contenente gli Studi in onore di Francesco Cataluccio (William Godwin ed il suo Journal all'epoca della Rivoluzione Francese, xv, 1984), e poi su Studi settecenteschi (Il governo più semplice. Il mito democratico-repubblicano in William Godwin, viII, 1987). Un terzo ampio articolo, che contiene spunti davvero interessanti sul romanzo utopistico di Godwin, rimase inedito, sino a oggi, malgrado l'oggettivo e rilevante valore del contributo di Farinella. Crediamo, pertanto, di fare cosa gradita alla memoria dell'amico e dello studioso pubblicandolo per la prima volta, in questa sede. ${ }^{1}$

Historian with multiple interests, librarian, musician, man of culture and great modernist, scholar, in particular, of Eighteenth-Century Italy, Calogero Farinella prematurely

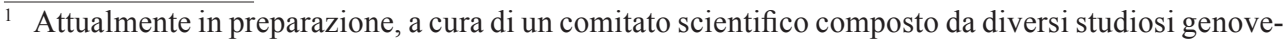
si e insubri, la raccolta completa degli scritti di Calogero Farinella, a eccezione dell'unica monografia da lui scritta, L'accademia repubblicana. La Società dei Quaranta e Anton Mario Lorgna, Milano, Franco Angeli, 1993, tratta dalla tesi di dottorato discussa a Venezia con Marino Berengo. Per quel che riguarda i criteri di edizione di questo inedito, i curatori hanno trascritto fedelmente il dattiloscritto originale, integrando solo alla fine la bibliografia con una scelta della letteratura più recente sul tema, nell'ultima nota a piè pagina. Le traduzioni dall'inglese sono originali dell'autore, verificate dai curatori. Solo di recente si è avuta una traduzione integrale del vasto e complesso romanzo utopistico di Godwin 2018. La prima traduzione molto parziale era Godwin 1912 (anonima, con illustrazioni di Osvaldo Tofani). Edizione parziale in italiano, col curioso sottotitolo «Il primo romanzo giallo della storia» (cosa peraltro non corrispondente al vero) è GodwIN 1976. Con il suo anarchismo di sinistra libertario ma alla Bakunin, visto che la società utopistica prevista da Godwin includeva sì l'abolizione o riduzione ai minimi termini dello stato, ma anche la completa eliminazione della proprietà privata Godwin destò un certo interesse nell'Italia degli anni Sessanta-Ottanta, dato il clima politico di allora; contemporaneamente agli scritti di Farinella - ma portiamo un solo esempio - veniva pubblicata la monografia di Di PIAZZA 1983 e numerosi articoli, sia in ambito anglistico, sia nell'ambito della storia moderna e della storia delle dottrine politiche [N.d.C.]
} 
left us in June 2019, at just over sixty years old. His publications on eighteenth-century Genoa and Enlightenment science (Verona especially: Francesco Bianchini, Anton Mario Lorgna and the Accademia dei Quaranta) make and will always give in the studies of history of culture. A student of salvatore Rotta (1926-2001), from whom he muttered his passion for the 18th century in particular, Farinella graduated under his guidance on William Godwin (1756-1836), father of Mary Shelley, radical philosopher and political writer of the libertarian area, who, with his fundamental work, marks the passage, in Britain, from the Enlightenment to Romanticism. On Godwin's figure and writings, obtaining them from his dissertation of his degree, Farinella extracted and published in the Eighties of the last century two articles, appeared in the volume of the Miscellanea storica ligure containing many studies in honor of the Professor Francesco Cataluccio (William Godwin ed il suo Journal all'epoca della Rivoluzione Francese, xv, 1984) and later on Studi settecenteschi (Il governo più semplice. Il mito democratico-repubblicano in William Godwin, viI, 1987). A third article, which contains really interesting ideas about Godwin's utopian novel, remained unpublished, to this day, despite the objective and relevant value of Farinella's contribution. We therefore believe that we are doing something that is pleasing to the memory of our friend and scholar by publishing it here for the first time.

\section{I legami con la POLITICAL JUSTICE}

Pochi giorni dopo la pubblicazione della sua opera principale, l'Enquiry Concerning Political Justice, avvenuta nel febbraio 1793, il filosofo e romanziere radicale inglese William Godwin (1756-1836) cominciò a scrivere un romanzo, Things Are They Are, or the Adventures of Caleb Williams, tutto pervaso dallo stesso fervente stato d'animo, con il quale aveva proceduto alla redazione della sua opera filosofica. La coscienza di avere mostrato al mondo le cause effettive del persistere di pregiudizi e pre-concetti e di avere additato i modi per superare i mali che sono la causa dell'infelicità dell'umanità, e così giungere a una nuova società, basata sui principi dettati dalla ragione, dalla verità, dalla giustizia, spinse Godwin a cercare di far conoscere a un pubblico quanto più ampio possibile quei principi, dato che, per lui, era essenziale che si iniziasse a discutere dei temi che aveva trattato nella Political Justice, affinché potesse avverarsi l'auspicato cambiamento della società, britannica e non solo.

Le due opere, pertanto, sono percorse da un intimo legame che le accomuna, collegate tra di loro dall'esposizione di temi e situazioni simili, formano un blocco abbastanza omogeneo e si illuminano e chiariscono vicendevolmente, forse proprio perché così diverse: la prima una trattazione e filosofica e politico-morale, la seconda un lavoro della fantasia, un libro di "avventure immaginarie". In tale modo, la denuncia e la critica sociale e politica della società e della sua struttura, che sono alla base della Political Justice, si ritrovano anche nel romanzo, politicità sottolineata da Godwin nella prefazione, che doveva accompagnare l'uscita del romanzo, nel 1794, ma che com- 
parve con la seconda edizione l'anno successivo, a causa della paura dell'editore che essa potesse essere oggetto della censura governativa. Il romanzo, spiega Godwin, è

[...] destinato a rispondere a uno scopo più generale e importante di quanto non appaia immediatamente a prima vista. Attualmente, il problema che domina nei confronti delle cose come esse sono, è il più interessante che possa presentarsi alla mente umana. Mentre una parte della società si richiama alla riforma e al cambiamento, l'altra esalta con i termini più accesi l'organizzazione sociale esistente. Sembrava dunque che si sarebbe tratto giovamento, per decidere su questo problema, se si fossero resi manifesti tutti gli effetti pratici di quell'organizzazione. Ciò che è qui presentato al pubblico non è una riflessione raffinata ed astratta; è uno studio ed una descrizione delle cose che accadono nel mondo morale. Solamente da poco tempo è stata riconosciuta l'importanza, inestimabile, dei principi della politica. È ora noto ai filosofi che lo spirito e l'essenza del governo si introducono in modo arbitrario in ogni rango della società. Ma questa è una verità quanto mai meritevole di essere portata a conoscenza anche di persone che presumibilmente non hanno occasione di leggere libri di scienza e di filosofia. Di conseguenza, ci si propose, nell'elaborare il presente lavoro, di delineare [...] un esame generale di un dispotismo familiare e mai documentato, tramite cui l'uomo diventa il distruttore dell'uomo. ${ }^{2}$

Mi è sembrato necessario riportare questa lunga citazione, per mostrare come il romanzo venga inteso, senza mezzi termini, quale uno strumento propagandistico, capace di fare penetrare principi e convinzioni politico-morali, un mezzo per disseminare verità, già scoperte e note. Essenzialmente, il Godwin vuole richiamare l'attenzione su quello che è il nucleo della Political Justice, ossia gli effetti terrificanti e nocivi, per l'umanità, prodotti dall'esistenza dei governi e di tutti gli apparati istituzionali che li accompagnano: la formazione di caste privilegiate e di interessi particolari che si contrappongono a quelli, più generali, dell'umanità; una amministrazione della giustizia organizzata in maniera tale da favorire i nobili e i ricchi, tutta tesa a difendere la proprietà privata e a punire quelle classi che nulla possiedono; l'importanza smodata accordata all'ineguaglianza, al lusso, allo splendore, che non è una cosa accidentale, ma è l'essenza dei sistemi politici aristocratici e monarchici, e che corrompe e travia e il giudizio e l'intelletto degli uomini, portandoli a favorire ed a desiderare la ricchezza e l'esteriorità, non il reale merito di uomini e cose.

Queste erano osservazioni in parte già fatte da altri pensatori come Rousseau, Helvétius, Bentham stesso: ma, a differenza di questi, e di altri, Godwin nega completamente che lo Stato possa continuare ad avere una funzione positiva per l'uomo, se mai l'ha avuta. Non è possibile continuare a mantenere una struttura che ha favorito guerre, dolori, distruzioni, assassinii, povertà, e sfruttamento: gli uomini si sono illusi che lo Stato fosse utile per mantenere in una situazione di equilibrio la società, garantendo la sicurezza ed il diritto, per dirla con Locke, alla libertà, alla vita ed alla proprietà. Il governo, pertanto, si fonda su questa erronea e falsa idea, che, se venisse distrutta, porterebbe alla caduta ed al crollo dello Stato e di tutte le costrizioni fisiche e morali, delle punizioni e delle restrizioni della propria libertà, che il presunto patto sociale, alla base della formazione dello Stato e della divisione tra i governanti e i governati, ha sempre comportato. L'uomo ha, finora, abdicato ad altri il proprio

\footnotetext{
$\overline{\overline{2} \text { Godwin 1970, p. } 1 .}$
} 
giudizio, la direzione delle proprie azioni, ma ciò è stato un male, e ha prodotto l'infelicità in generale. È, quindi, giunto il momento, secondo Godwin, in cui ogni uomo cominci a pensare e a decidere da sé, non demandi ad altri le proprie responsabilità, e segua una condotta che si adegui liberamente ai dettati della moralità, ai doveri che derivano dalla verità e dalla giustizia. Così, Godwin prospetta e delinea una società, in cui ciascuno prenda parte alle decisioni, intervenendo di persona, adoperando sempre il proprio intelletto, giudicando con la propria mente, senza che altri impongano giudizi o comportamenti da adottare: così le strutture del governo saranno ridotte al minimo, all'essenziale, la proprietà scomparirebbe e ciascuno avrebbe secondo l'autore il necessario per vivere.

Il romanzo di Godwin illustra, quindi, il funzionamento delle strutture sociali del suo tempo, e le conseguenze che ricchezza, egoismo, giudizi basati su codici morali che non si richiamano alla moralità e alla giustizia producono. Alla base del romanzo c'è, a mio parere, l'illustrazione della definizione di "giustizia" che Godwin aveva dato nella Political Justice: «se il termine giustizia ha qualche significato, esso si situa proprio nel fatto che io dovrei cooperare a tutto ciò che è in mio potere di fare in vista del beneficio del tutto. [...] La giustizia è una regola di condotta, che ha origine nella connessione di un essere percipiente con un altro». ${ }^{3}$

Essa è dunque un trattamento imparziale, e non distorto da errori e da pregiudizi che un uomo deve adottare nei confronti di un altro uomo e della società tutta. Ora, è proprio la mancata adozione di tale rapporto imparziale che viene illustrata nel romanzo godwiniano, e produce tutta l'azione: i due personaggi principali non si adeguano a tale regola e, da ciò, nasce tutta la loro infelicità, le loro incomprensioni, la persecuzione reciproca, proprio perché nessuno dei due ha tentato di uscire dal suo egoismo, dai suoi pregiudizi: ma il dovere morale induce ad agire diversamente, non considerando il proprio beneficio, bensì quello generale dell'umanità - l'atteggiamento di Godwin, che richiama quello di Kant e dell'Illuminismo più in generale. La virtù richiede che ciascuno si ponga «al posto di uno spettatore imparziale, si supponga di natura angelica, osservandoci da un luogo elevato, non influenzati dai nostri pregiudizi». ${ }^{4}$ Se non si agisce così, se non si seguono questa regola e questo comportamento, si va verso la distruzione, si soffre, e «l'uomo diventa il distruttore dell'uomo», come di se stesso.

Nel romanzo, Caleb Williams viene assunto come segretario dal nobile e umbratile Falkland, il cui carattere ritirato è completamente differente da quello che aveva mostrato precedentemente. Un fittavolo di Falkland, Collins, narra a Caleb la giovinezza e l'educazione del padrone, descrive il Grand Tour da lui compiuto in Francia e in Italia, la cortesia e la gentilezza che lo rendono l'idolo di tutti gli uomini e di tutte le donne che lo conoscono, così come l'alta ed esagerata concezione dell'onore, che egli ha. Tornato dal continente, Falkland si stabilisce nella sua contea, dove si trova già il prepotente e nobile Tyrrel, dal carattere opposto: è volgare, incolto, di gusti rozzi, dedito alla caccia, al bere e alle zuffe. L'arrivo di Falkland, che si fa il paladino di tutti

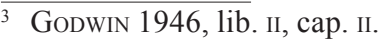

4 Ibidem.
} 
coloro che subiscono le ingiustizie di Tyrrel, muta radicalmente la vita sociale nella contea: mentre prima Tyrrel dettava legge ed era rispettato anche per il terrore che incuteva, ora è sempre più escluso e Falkland, al contrario, è adulato, corteggiato, conteso. L'attrito tra i due, per la gelosia di Tyrrel, cresce sempre più, malgrado gli sforzi di Falkland di trovare un accordo con l'altro.

Il loro rapporto è complicato dalla devozione-amore che Emily, la cugina povera di Tyrrel, prova nei confronti di Falkland. Tyrrel cerca e in ogni maniera di impedire ai due di incontrarsi, sino poi a perseguitare spietatamente Emily, e a mandarla in prigione, dove, a causa degli stenti, ella muore poco dopo. Falkland si reca allora da Tyrrel e gli comunica che per la sua crudeltà non troverà più pace, dato che egli lo denuncerà pubblicamente ogni volta che lo vedrà.

Una sera, a un ballo, Falkland mantiene la sua promessa: Tyrrel, sommerso dalle accuse e dallo sdegno generale, abbandona la festa, ma vi ritorna poco, dopo colmo d'ira, deciso a vendicarsi. Con alcuni pugni, manda a terra il rivale e lo spinge, a calci, per la sala. Ciò è un colpo mortale, per la concezione dell'onore di Falkland, una macchia che non potrà essere mai più tolta. E, la stessa notte, Tyrrel viene trovato ucciso da una coltellata; il sospetto dell'omicidio ricade su Falkland, il quale però, ricordando la sua vita e le sue concezioni, convince tutti che non può essere lui il colpevole. Vengono accusati e condannati a morte gli Hawkins, padre e figlio, ex fattori di Tyrrel, dal quale avevano subito molti soprusi. Tuttavia, da quel momento, il carattere di Falkland muta, diviene molto più triste e tetro, si apparta e vive come nell' angoscia.

Caleb è attratto dal mistero che emana da Falkland e la sua curiosità si accende. Scopre, così, che Falkland conserva, gelosamente, un cassettone, che, preso da un'insaziabile e spasmodica voglia di sapere, forza: mentre sta frugando tra il materiale racchiusovi - le prove della colpevolezza di Falkland - viene scoperto dal padrone. Caleb sospetta, è quasi certo, che Falkland sia l'uccisore di Tyrrel e abbia lasciato condannare ingiustamente gli Hawkins, e ricerca, con mille allusioni e sotterfugi, di fare cadere Falkland in fallo e di costringerlo a tradirsi. Terrorizzato, Falkland asseconda la diabolica curiosità di Caleb e gli confida che lui è il vero assassino di Tyrrel, ma avverte il giovane che non potrà lasciarlo mai più, perché non può permettere che il suo onore sia messo in pericolo da una possibile confessione di Caleb ad altri del suo segreto. Caleb promette di non svelare a nessuno quanto gli ha riferito, tuttavia la sua vita diviene un inferno: sempre spiato da Falkland, sospettato di volere comunicare la verità, a chiunque gli si avvicini. Stanco di questa situazione, Caleb non resiste e fugge dal padrone-oppressore. Viene però preso e ricondotto da Falkland, perché accusato di un furto non commesso. Caleb è portato così in prigione, dove conosce le sofferenze e l'abiezione morale, prova l'iniquità di una società che condanna gli innocenti. Riesce a scappare una prima volta, ma non fa in tempo ad assaporare la libertà che viene ripreso immediatamente. Fugge una seconda volta e, nella fuga, egli è torturato dall'ansia di doversi nascondere a nemici potenti, di sentirsi oggetto della persecuzione e sapersi braccato. 
Nelle sue peregrinazioni, ha modo di unirsi a una banda di ladri il cui capo, Raymond, si presenta come un uomo che cerca di attuare una giustizia alternativa e più vera di quella che va a solo vantaggio di ricchi e nobili. Però, ciò avviene tramite la ribellione e la contestazione violenta, l'auto-esclusione dalla società. Caleb non condivide questa condizione e si allontana così dalla banda. Intanto, l'apparato repressivo giudiziario è rivolto contro di lui: ricercato in tutto il paese, circolano opuscoli che narrano la sua vita in modo deformato, descrivendolo come un feroce malfattore, omicida, il capo di una banda sanguinaria.

Si reca a Londra, oramai costantemente seguito da Gines, un ladro assoldato da Falkland, perché possa impedire a Caleb di potere stabilire rapporti di fiducia con alcuno. Caleb si guadagna da vivere scrivendo storie di pirati e banditi: tramite questo indizio, Gines scopre il suo nascondiglio e così Caleb deve riprendere a vagare per l'Inghilterra. Cerca di ottenere fiducia dai giudici, ma i suoi tentativi, dato che rifiuta, ostinatamente, di svelare, chiaramente, la colpa di Falkland, falliscono; il rango, l'onore e la ricchezza hanno più importanza della verità. Deluso, deciso a ritirarsi dal mondo, Caleb cerca scampo in un remotissimo villaggio gallese, lontano dalla società, quale egli l'ha conosciuta, ove sembra potere ritrovare la pace interiore, la tranquillità e un fiducioso rapporto con tutti gli altri esseri umani, che le circostanze gli avevano impedito di conoscere. Si introduce nella piccola comunità e stringe amicizia con molti, tra cui Laura, una madre di famiglia, semplice, candida e intelligente. Ma anche qui arriva Gines, che fa circolare l'opuscolo, che narra la truce storia di Caleb, cosicché il villaggio lo rifiuta: il suo tentativo di spiegare la verità a Laura non ha luogo, poiché ella, sentendosi tradita, rifiuta persino di ascoltarlo. Caleb, allora, è al colmo della sopportazione: decide di liberarsi del fardello per il quale ha dovuto affrontare persecuzioni e angherie, e di confessare la colpa di Falkland davanti ai giudici. Il giorno del processo, la sua intenzione di scagliarsi contro Falkland, di ottenere finalmente giustizia, il suo impeto battagliero, crollano di fronte a sé: trova un Falkland malato, cadente, prossimo alla morte. Pronuncia, allora, un discorso, con il quale ammette sì la colpa di Falkland, ma soprattutto riconosce e sottolinea che lui, Caleb, ha rovinato l'esistenza di un suo simile, di un altro uomo. Commosso dalle sue parole, Falkland confessa l'omicidio commesso e dichiara la sua ammirazione per Caleb gettandosi tra le sue braccia. I due riconoscono reciprocamente di essere l'uno la causa dell'infelicità dell'altro e si chiedono perdono. Pochi giorni dopo, Falkland muore e Caleb conclude il suo racconto sottolineando il rimorso e il tormento, che lo perseguiteranno per essere stato la maggiore causa e dei dolori e della morte di Falkland.

Come quasi tutti i romanzi editi tra la fine del Settecento e gli inizi del primo Ottocento, il Caleb Williams venne pubblicato in tre libri: Godwin, tuttavia, riuscì a fare di questa necessità, tipografica ed editoriale, di questa costrizione esterna, la parte essenziale del romanzo, la sua struttura fondamentale, che detta, tanto la trama, quanto il clima stesso della narrazione. Il romanzo è, infatti, divisibile in tre grandi parti: la descrizione dell'educazione e delle qualità raffinate del nobile Falkland e del brillante mondo sociale da lui frequentato; il mutarsi di Caleb nell'instancabile persecutore di 
Falkland al fine di scoprirne il segreto, scrutandolo per infine sorprenderlo, riempiendolo di terrore e angoscia; infine, la persecuzione verso Caleb da parte di Falkland, che induce il primo a fuggire, a nascondersi, a vagare, preda a sua volta dell'angoscia e inseguito dai sicari, dalla legge, dai pregiudizi umani.

Il parossismo, la frenetica concitazione, l'atmosfera di persecuzione e di incombente tragicità sono tra i protagonisti del romanzo, tensione che si accumula, pagina dopo pagina, favorita dalla stessa struttura della narrazione, in attesa di uno scioglimento definitivo, che sembra però non arrivare mai: una struttura quasi binaria, in cui i capitoli si susseguono in modo tale da alternare, efficacemente, il clima di tranquillità e quello di disperazione, così che, alla speranza, fanno seguito l'angoscia e il terrore, che a loro volta lasciano il posto alla quiete e alla pace.

Del resto, questa tragicità e questo clima di persecuzione sono accentuati dalla costante presenza nel romanzo di tetre prigioni, di corti di giustizia, di condanne, di problemi connessi alla legislazione criminale: e non a caso il romanzo si conclude in un'aula di tribunale. È il riflesso del grande dibattito illuminista che almeno da Beccaria (1764) in poi aveva acceso la discussione in Europa sul diritto dello Stato di punire i rei. È qui evidente che Godwin vuole così illustrare la propria posizione di assoluta contestazione di tale diritto, perché vi è non solo il rischio, date le attuali istituzioni, che in prigione vadano gli innocenti, più che i colpevoli, ma essenzialmente perché, se lo scopo delle punizioni e delle prigioni è la riforma dell'uomo, questi strumenti non la favoriscono: l'uomo è governato dalla ragione; pertanto, in ogni campo, deve prevalere l'appello «al tribunale della propria mente». Gli argomenti sì possono convincere, la persecuzione no, anzi l'appello alla forza, a ciò che non è né ragione né verità, mina alla base il concetto stesso di giustizia e di verità, perché fa della costrizione e della violenza il solo metro per giudicare la condotta di un altro uomo. ${ }^{5}$

\section{IL FINALE DEL ROMANZO E IL SUO MESSAGGIO POLITICO}

Tuttavia, mi sembra che nel romanzo vi sia qualcosa di più e di diverso di un pedissequo quanto costante richiamo alle teorie espresse da Godwin nella Political Justice, o perlomeno esso è più sottile. Per comprendere, appieno, il messaggio del romanzo, a mio avviso è necessario partire dal finale, dato che questo illumina tutte le vicende precedenti, ne fornisce insomma la chiave, per una possibile linea interpretativa: non a caso, anche materialmente, il finale è posto al fuori della tripartizione del romanzo godwiniano fatta notare in precedenza. La suddivisione in capitoli si arresta e la scena ultima di Caleb e Folkland che si incontrano e si abbracciano è narrata in un "poscritto", cioè è posta in una posizione che ne sottolinea l'importanza, il significato, la rilevanza.

Il problema di come finire il romanzo si pose seriamente a Godwin: infatti, del finale esistono due versioni differenti. Il finale originario prevedeva che Caleb, giunto al colmo della sopportazione, stanco di affrontare le persecuzioni, presa la decisione

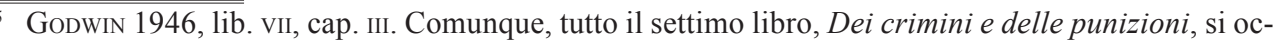
cupa di questi problemi.
} 
di confessare la colpa di Falkland, davanti ai giudici, una volta giunto in tribunale, si scagliasse contro Falkland, senza alcuna pietà, né rimorsi, e che questi contrastasse, altrettanto vigorosamente, le affermazioni dell'avversario: lo status sociale, il favore cioè accordato dal sistema giudiziario a chi era nobile avevano il sopravvento e Caleb, non creduto, veniva condannato alla prigione e finiva i suoi giorni incatenato, vittima sacrificale, sull'altare delle "cose così come sono". Ma questa conclusione evidentemente - non dovette soddisfare Godwin, che sentiva la necessità di adottare una "nuova catastrofe" (in senso aristotelico). Dal 4 all' 8 aprile 1794, dunque, egli ne scrisse un'altra, la definitiva, quella che vede premiare il rimorso di Caleb, da me riassunto poco più sopra.

Il problema del doppio finale ha posto alcuni interrogativi ai critici, portandoli, anche, ad alcuni travisamenti. Secondo alcuni, il nuovo finale - il definitivo - rappresentava la vittoria di Caleb, perché costringe Falkland a confessare le sue colpe e a riconoscere l'innocenza del primo. Inoltre, malgrado il rimorso di Caleb, «il significato del finale rivisto è ottimistico: la Verità può prevalere, perfino contro le forze associate di quelli che sostengono le cose così come sono». ${ }^{6}$

Per altri, poi, la nuova conclusione sarebbe in aperta contraddizione con alcune affermazioni della Political Justice, come la necessità che si discuta a lungo, affinché la verità sia riconosciuta e adottata come una regola di comportamento, da ciascuno, mentre in particolare il riconoscimento dell'innocenza di Caleb contrasterebbe con il ferreo determinismo godwiniano, in base al quale non potrebbe esserci un così improvviso riconoscimento, poiché ogni azione non ne segue un'altra, autonomamente, dalla catena dei fatti precedenti, ma risulta strettamente determinata dal tipo di circostanze che la precedettero. ${ }^{7}$ È proprio vero che la conclusione scartata veda la sconfitta di Caleb, mentre quella definitiva rappresenterebbe un finale ottimistico e più fiducioso? Il profondo rimorso che si appropria dell'animo di Caleb è solo una mitigazione della sua vittoria, altrimenti troppo piena? Godwin si discosta e così profondamente dal principio della Political Justice?

Il nuovo e definitivo finale conferma, mi sembra, il contenuto ideologico di fondo del romanzo: in una società strutturata secondo la concezione dell'onore e dei rapporti interumani, che tutto concede all'esteriorità e nulla all'essenza intima dell'uomo, dove esistono il rispetto e il predominio della ricchezza e delle caste privilegiate, non solo non è possibile ottenere giustizia né il riconoscimento vero e profondo, non accidentale o casuale, della verità, ma tutti, colpevoli e innocenti, sono destinati alla

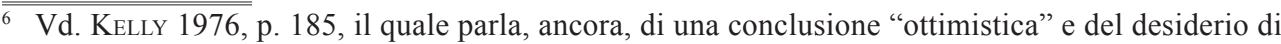
evitare una conclusione pessimistica (ivi, p. 190). Cfr. anche Fleischer 1951, che, incidentalmente (p. 25), afferma che nel finale, spinto ormai oltre limite a cercare la rovina e l'infelicità del criminale Falkland ed essendovi riuscito, Caleb sarebbe sopraffatto al colmo del proprio trionfo dal rimorso per il disastro che ha portato a termine.

7 Vd. Dumas 1966, pp. 575-597, che afferma anch'egli che il finale definitivo sarebbe meno consono dell'altro allo scopo ideologico generale del romanzo e della Political Justice. Su quest'ultima, cfr. RotTA 1981, pp. 176-177.
} 
comune distruzione e nessuno può sperare di potere così raggiungere la felicità, per quanto limitata e individuale essa possa essere.

Il finale scelto da Godwin non rappresenta - a mio avviso - la vittoria di Caleb, il suo "trionfo definitivo", perché esso maschera e spunta ogni tentativo di Falkland di occultare la verità, vedendosi riconoscere l'innocenza proprio dal malvagio persecutore. La vittoria di Caleb era, invece, nel finale scartato: era qui il suo trionfo di fronte alle "cose come esse sono". Perseguitato ingiustamente, davvero innocente, era sottomesso dall'organizzazione sociale, dai privilegiati che continuavano a perpetuare in maniera egoistica lo stato di ingiustizia, a loro beneficio, ma, proprio così, Caleb si "salvava", perché subiva il martirio nella testimonianza della verità, era imprigionato a causa della sua innocenza, era in altre parole il testimone-vittima dell'iniquità delle strutture politico-sociali, che così uscivano davvero e definitivamente condannate, e identificate con l'oppressione e la corruzione: le colpe di Caleb erano "perdonate" tramite l'ingiusta condanna, mentre, in questo modo, la sua ribellione nei confronti del padrone-oppressore sarebbe stata giustificata, così come sarebbe stata giustificata qualsiasi ribellione nei confronti dei meccanismi sociali, corrotti, non riformabili per gradi, perché l'uomo avrebbe dovuto sottomettersi supinamente a essi, oppure cambiarli violentemente e radicalmente: dato quel finale, una soluzione diversa non poteva essere prospettata. Questa "salvezza" morale di Caleb sarebbe stata totale e incondizionata e avrebbe, così, sublimato e portato a redenzione le ingiustizie sofferte dagli altri personaggi - gli Hawkins, Raymond, Emily - proprio tramite il sacrificio finale di Caleb, con quella richiesta di giustizia insoddisfatta, vilipesa e condannata a nuove sofferenze.

Al contrario, con il nuovo finale, Godwin pone l'accento sul fatto che nessuno, nelle strutture sociali date, si può salvare se le istituzioni e gli uomini continueranno ad essere quali essi sono, succubi e delle passioni e della gelosia. Ogni cosa è, invece, destinata a perire, a subire una condanna e una sconfitta, definitiva e totale, perché l'ingiustizia corrode, alla base, tutti i rapporti umani, la distruzione incombe e infesta ogni uomo, mentre la ricerca della felicità diventa impossibile, e è negata. Le parti contrapposte della società si scontrano e in questo scontro nessuno trova salvezza, bensì l'una parte diventa la rovina dell'altra: si distruggono a vicenda.

La malattia di Falkland e la sua morte, dopo la conclusione del processo, così come il rimorso che viene a impossessarsi dell'anima di Caleb, la consapevolezza di essere infatti il colpevole della fine dell'altro, rimorso che lo perseguiterà per tutta la vita futura, impedendogli di poter raggiungere quello per cui Caleb aveva sopportato ogni prevaricazione e ogni persecuzione, ovvero una vita normale, dimostrano la condanna-distruzione reciproca che non lascia sul campo eroi vittoriosi ma solo sconfitti: collettivamente le istituzioni e la società; individualmente Caleb, Falkland e gli altri personaggi. Si vedano, a conferma di ciò, le considerazioni di Caleb che chiudono il romanzo:

Venni qui per maledire, ma ora non mi resta che benedire. Venni per accusare, ma sora sono spinto ad applaudire. Io dichiaro a tutto il mondo che Mister Falkland è un uomo degno e di 
affetto e di benevolenza, e che io stesso sono il più indegno ed il più odioso essere umano. Non mi perdonerò mai l'ingiustizia di questo giorno. Il ricordo mi perseguiterà, e renderà amara ogni ora della mia esistenza. Agendo così, mi sono dimostrato un assassino, un freddo, deliberato ed insensibile assassino. [...] La morte sarebbe un favore a confronto di ciò che provo. Tali erano gli accenti dal mio rimorso dettati. Li espressi con una impetuosità incontrollabile, poiché il mio cuore era molto commosso e io mi sentivo costretto a dare sfogo alla sua angoscia. [...] Ricordo le lodi che Mister Falkland mi rivolse, non perché le meriti, ma perché esse servono ad aggravare la bassezza della mia crudeltà. Egli sopravvisse a questa terribile scena solo tre giorni. Io sono stato purtroppo il suo assassino. [...] Quale atroce, esecrabile, indegna persona mi sono mostrato. Gli ho deliberatamente inflitto un'angoscia mille volte peggiore della morte. [...] Ecco quale è il risultato di un progetto che delineai per liberarmi dai mali che mi hanno seguito, per così lungo tempo. Pensavo che se Mr. Falkland fosse morto, io sarei tornato ancora una volta a gustare tutto ciò che rende la vita degna di essere vissuta. Pensavo che, se fosse stata riconosciuta la colpa di Falkland, la fortuna e il mondo avrebbero sorriso alle mie azioni. Ambedue questi eventi si sono avverati ed è solo adesso che sono veramente infelice. ${ }^{8}$

Ciò che «rende la vita degna di essere vissuta», ormai, proprio quando sembrava raggiungibile ed a portata di mano, è inarrivabile, non potrà esserci alcuna vita da vivere, per Caleb, e ciò malgrado il riconoscimento della verità che allora, oltre a essere quanto mai amaro, è soprattutto formale, esteriore poiché non produrrà alcun mutamento nei rapporti sociali, né negli individui, come invece il Godwin asseriva nella Political Justice, ove affermava che bastava che la verità fosse svelata, perché gli uomini si adeguassero alle sue leggi e mutassero i comportamenti, abitudini, modi di pensare: «la verità è certo onnipotente, $[. .$.$] e quando viene comunicata in modo ade-$ guato è irresistibile, per ciò che ha attinenza con la ragione umana. Ogni proposizione che può essere illustrata alla mente produrrà infallibilmente un effetto, corrispondente, nel comportamento». Ancora: «La proprietà della verità è di propagarsi. [...] Le cause che sospendono il suo progresso derivano [...] dalla guardinga ed intollerante gelosia, propria dei governi tirannici. [...] È necessario che la verità venga comunicata. [...] Non c'è premio migliore che si possa accordare alla virtù eminente più che questo: la chiara e semplice proclamazione della sua eccellenza, di fronte a tutto il mondo» ${ }^{9}$. Di fronte a queste chiare affermazioni, la proclamazione della innocenza di Caleb, il ristabilimento della verità nel romanzo passano appunto in secondo piano, come messi da parte, non sono importanti; diventano elemento non essenziale, di fronte all'angoscia, al mero rimorso, al dolore e pentimento, che si appropriano di lui. Il finale del romanzo è come sospeso, quasi inesistente, domina il senso di non-conclusione: il delitto è realmente punito? La virtù riceve la giusta e meritata ricompensa? Le risposte sono negative e affermative allo stesso tempo, anzi non v'è risposta, domina solo un doloroso silenzio.

La differenza con i romanzi successivi di Godwin è evidente, poiché tutti terminano chiaramente e senza alcuna ambiguità nel più canonico dei modi: la ricompensa dell'eroe. Godwin conclude il Saint Leon. A Tale of the Sixteenth Century (1799) con parole che si ricollegano al Caleb Williams in maniera diretta: «Qualunque possa es-

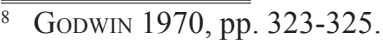

9 Godwin 1946, lib. I, cap. v; lib. I, cap. vi; lib. vI, cap. vI.
} 
sere stato il risultato della mia personale esperienza della vita umana, io non posso mai ricordare il destino di Carlo e Pandora, senza confessare con esultanza che questo nostro indaffarato ed ansioso mondo ha in serbo, malgrado tutto, qualcosa per il quale sia degno vivere». ${ }^{10}$

Nel 1799, la riconciliazione dell'eroe con il mondo, la possibilità di stabilire un compromesso, con una società dalle strutture ingiuste, e, così, nonostante tutto, continuare ad affrontare la vita, appare essere possibile: nel 1794 no. È una conclusione quanto mai pessimistica, quella del Caleb Williams, abbastanza distante, a prima vista, dalla Political Justice, con la sua fede quasi cieca e molto fiduciosa nell'avvento del regno della verità, che gli uomini necessariamente venereranno. Invece, il pessimismo è in parte mitigato se si pensa all'implicito, ma non troppo, richiamo a un'eguaglianza di fondo di tutti gli uomini, a una comune umanità, che accomuna tutti e che deve rimandare a una sincera solidarietà umana: ecco, quindi, che soltanto se si è consci che lo stato attuale della società e l'ineguaglianza delle condizioni permettono la distruzione di tutta la società, nel suo complesso, ove nessuno potrà mai dirsi vittorioso, solo se si è consapevoli di questo, allora, si potrà mutare e cercare di perseguire la felicità. Soltanto questo cambiamento profondo dei cuori potrà permettere dunque la rigenerazione e la salvezza comune del genere umano.

Il messaggio del romanzo si concretizza, dunque, proprio nel rimorso finale di Caleb, e le sue disgrazie, tutta la sua vicenda, divengono una sorta di avvertimento: non si deve perseguitare un essere umano, facendogli sentire l'affanno della persecuzione, della denuncia, della rovina, costringendolo ad appellarsi ai sentimenti più bassi e disumani e a trasformarsi, a sua volta, in persecutore implacabile, invece di affidarsi alla discussione, invece di fidarsi della sua umanità e di avere la totale fiducia nelle conseguenze della più completa sincerità. È Caleb a pronunciare le seguenti parole, che valgono, tanto per lui, quanto per Falkland:

Adesso vedo quell'errore in tutta la sua enormità. Sono sicuro che, se avessi aperto il mio
cuore a Mister Falkland, se avessi raccontato a lui solo quello che ora sto dicendo, egli non
avrebbe potuto resistere alla mia ragionevole domanda. [...] In ogni caso, se egli doveva es-
sere in mio potere, in quale modo avrebbe potuto cercare la sua salvezza, tentando di ricon-
ciliarsi con me o ricorrendo a una crudeltà inesorabile? Mister Falkland è di nobile natura.
È quindi impossibile che avrebbe potuto rifiutare una franca e fervente spiegazione, la fran-
chezza e il fervore che ricolmavano la sua anima. Mi lasciai vincere dalla disperazione, men-
tre c'era ancora tempo per trovare la maniera giusta; ma la mia disperazione fu criminale, fu
un tradimento verso la sovranità della verità. ${ }^{11}$

L'innocenza di Caleb e la dichiarazione finale, con la quale la verità viene ristabilita, sono minate all'interno, infette da qualcosa, che non fa parte dell'innocenza, né della verità stessa; per questo, esse passano in secondo piano, come se il loro riconoscimento non fosse avvenuto. Non vi è scostamento dalla Political Justice: ove predominano

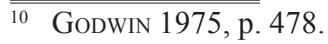

11 Godwin 1970, p. 232.
} 
il sospetto, l'insincerità, la paura, il terrore, la non discussione, là c'è solo il rimorso, l'infelicità, la distruzione, la morte.

Infatti, Godwin aveva richiamato l'attenzione sull'importanza della sincerità, perché la relazione tra ciò che si pensa e le azioni esteriori dell'uomo non è solo alla base della sua concezione di moralità, ma è il solo mezzo tramite il quale l'umanità possa svilupparsi, ed arrivare alla piena razionalità: dire e sempre la verità è uno dei compiti che Godwin impone ai riformatori dell'uomo, ai filosofi, ai numerosi filantropi. ${ }^{12}$ Godwin, pertanto, non gioca qui sull'equivoco e sull'inter-scambiabilità tra l'innocenza e la colpevolezza, come afferma invece un critico, ${ }^{13}$ ma anzi sottolinea semmai come il non adottare i criteri e la condotta che discendono dalla verità e dalla sincerità provochi la distruzione. In Caleb ha infatti il sopravvento il rimorso, perché ha tradito la causa e della verità e della sincerità, sfiducia che è la sua colpa più grave e per la quale egli è come punito.

Insomma, il romanzo è un appello accorato, una sorta di ultima ratio, con cui Godwin richiama l'attenzione dei contemporanei, di fronte a una situazione storica che vedeva avvicinarsi lo scontro cruento tra radicali giacobini inglesi da un lato e il governo del giovane Pitt dall'altro, ormai avviato lungo la strada della repressione e del non-rispetto delle leggi, come avevano dimostrato la condanna in contumacia di Thomas Paine, i processi dei primi mesi del 1794, in Scozia, contro alcuni radicali e la soppressione dell'Habeas Corpus.

La tragicità del messaggio godwiniano si pone proprio in questo più che accorato appello alla ragione ed alla fiducia e sincerità, che non rinnega i principi della Political Justice, bensì li raccomanda: o si fanno prevalere le indicazioni suggerite in quell'opera - quindi non ci si scontra, ma si discute per arrivare ad una comprensione piena e solenne della verità, da parte di tutti, così che possa produrre dei frutti - oppure non vi è salvezza per nessuno, davanti all'intolleranza ed al sospetto generalizzato. Non vi sono per Godwin altre soluzioni o alterative. ${ }^{14}$

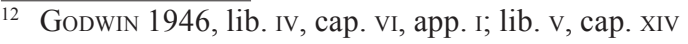

13 Cfr. Furbank 1955, p. 218.

14 Il passare del tempo, tuttavia, smorzava notevolmente la denuncia sociale e politica del romanzo, mentre veniva così a prevalere l'aspetto più strettamente psicologico, narrativo e inventivo: l'attenzione si sarebbe concentrata non più sulle "cose come esse sono", bensì sulle avventure di Caleb Williams. È lo stesso Godwin a compiere tale operazione quando diversi anni dopo, nel 1832, in un clima di trionfante Romanticismo, egli descrive la genesi di Things As They Are. Il romanzo, afferma Godwin, doveva distinguersi, per mezzo di un vigoroso e prepotente interesse, che catturasse, così, le attenzioni del lettore, producendo effetti duraturi e nella sua mente e sulla sua immaginazione, così che nessuno - dopo averlo letto - fosse esattamente lo stesso uomo che era stato prima della lettura del romanzo. Occorreva dunque mettere in rilievo lo sconvolgimento, il clima di attesa, l'imprevedibilità e il brivido. Per rafforzare questa idea, Godwin scrisse che dapprima delineò il soggetto del terzo volume, in cui immaginava la situazione di un uomo costretto a fuggire, e in preda al continuo terrore di essere sopraffatto dalle peggiori calamità, e l'inseguitore che, con la sua ingegnosità e le sue risorse, teneva la vittima in uno stato di terribile allarme. Il secondo libro doveva quindi servire a spiegare l'impulso che il persecutore sentiva verso la sua vittima, mostrando l'impellente motivo della persecuzione, che Godwin trovò in un omicidio commesso dall'inseguitore, mentre l'inseguito ne voleva scoprire il colpevole. Per ultimo, Godwin delineò il tema che doveva formare il materiale del primo volume,
} 


\section{LA SCISSIONE INTERIORE}

Comunque, mi sembra che nel romanzo non vi sia soltanto questa denuncia quasi disperata di una società lacerata in classi e interessi contrapposti. Godwin richiama l'attenzione sugli effetti di un'altra separazione, di un'altra tragica scissione. Infatti, Caleb Williams e Falkland sono, a mio avviso, due personaggi specularmente complementari, l'uno la proiezione diversificata dell'altro, che non potevano vivere, se non in funzione di reciprocità, completa e totale, per cui i due debbono stare insieme, perché formano un tutto completo e autosufficiente, mentre divisi e lontani, non comprendendosi, troveranno soltanto l'infelicità, l'incompletezza, e un sé diviso.

La fuga di Caleb dal mondo e dalla presenza del padrone, che trova il momento corrispondente nell' incomprensione di Falkland, riguardo le ragioni di Caleb, non è veramente tale, perché più gli sembra di allontanarsi da Falkland, e dal suo potere, più ne sente la presenza; fuga che diventa, pertanto, rincorsa reciproca tra i due personaggi del romanzo e la loro lotta, più che essere una autentica persecuzione, nasconde la ricerca dell'altro, una repulsione-attrazione che si risolve solamente nell'identificazione finale dei due, con quell'abbraccio che significa una sussunzione delle due parti, un finale riconoscersi, così come si riconosce la sconfitta reciproca finché i due rimangono separati: l'uno, Falkland, accettando un codice morale incondizionatamente, e sacrificando all'onore ogni altra considerazione, continuando a difenderlo, anche quando ciò porta alla malattia, all'auto-consunzione; 1'altro, Caleb, cercando di fuggire individualmente, con una soluzione personalistica della società e delle sue ingiustizie. Sia per Falkland, sia per Caleb, è importante e anzi fondamentale la ricerca della felicità, ma, proprio poiché ciascuno cerca di raggiungerla a suo modo, indipendentemente l'uno dall'altro, essa è infine irraggiungibile.

Insomma, Caleb rappresenta la parte "libera" e ingenua, naturale e innocente dell'uomo, che è naturalmente curiosa e affascinata dal mondo delle cose; mentre Falkland, dal canto suo, è, invece, la cultura non naturale, ma elaborata, la raffinatezza, il gusto per ciò che è esteticamente bello. I due, in definitiva, rappresentano il frutto della società, quale essa è nella Gran Bretagna del tardo Settecento: 1'alienazione umana, la separatezza, la divisione tra cultura e natura, tra soggettività e oggettività, tra il mondo interiore della coscienza e quello esteriore della realtà, con le sue leggi, che negano i rischi della coscienza umana stessa.

Il rimando, ovviamente, è a Rousseau, le cui opere Godwin aveva letto avidamente, con la sua condanna dell'alienazione dei rapporti umani e dei sentimenti, di una società e dei suoi codici morali, che impongono all'uomo di vivere fuori di sé, nell'opinione altrui, schiavo delle convenzioni, del denaro, delle ambizioni, degli altri uo-

in cui, per rendere più drammatico il successivo cambiamento, il persecutore e assassino veniva presentato «in un'atmosfera fantastica, cosicché ogni lettore dovesse sentirsi spinto, quasi, ad adorarlo per le altre sue qualità». Questo resoconto della nascita del romanzo venne scritto da Godwin a mo' di prefazione a una nuova edizione a stampa di un altro suo romanzo, Fleetwood or The New Man of Feeling (1805), una prefazione ora ripubblicata in GoDwin 1970, pp. 335-341. La citazione da me fatta si trova alle pp. 336-337. 
mini. Godwin, tuttavia, non condanna tutti gli aspetti della civiltà o la stessa cultura, anzi: è conscio del grado di civilizzazione al quale gli uomini sono giunti, per cui auspica che questi possano appropriarsi di tutti gli aspetti positivi, che il processo storico-sociale ha portato con sé e di conciliare la cultura con un bene essenziale - uno dei motori che può portare alla eguaglianza sociale - perché l'umanità possa progredire, e tramite la conoscenza e la discussione, così giungere a instaurare rapporti sociali e interumani che, al contrario di quelli esistenti, si fondino sul reale merito degli uomini e delle cose, sulle qualità vere e interiori dell'uomo e non sulle apparenze. ${ }^{15} \mathrm{Si}$ veda, ad esempio, il modo in cui Godwin presenta per la prima volta la figura di Falkland:

Ferdinando Falkland, un tempo, fra tutte le persone allegre, era il più allegro. Non certamente di quel frivolo tipo che suscita disprezzo invece che ammirazione, e la cui levità assomiglia semmai a sconsideratezza, più che a felicità. La sua allegria era sempre accompagnata da dignità. Era cioè la allegria dell'eroe e dello studioso, purificata dalla riflessione e dalla sensibilità e che mai perdeva di vista sia il buon gusto, sia la generosa bontà. Così come si mostrava, essa denotava una genuina gioia del cuore, che irradiava una inimmaginabile vivacità d'ingegno sulla sua compagnia e sulla sua conversazione e lo rendeva la continua delizia dei vari circoli che allora egli frequentava molto volentieri. ${ }^{16}$

\title{
Si confronti ora questa descrizione con il ritratto iniziale di Caleb:
}

\begin{abstract}
Non mi venne insegnata alcuna nozione di scienza, eccetto il leggere, lo scrivere e il far di conto. Ma avevo una mente curiosa, che non si negava nessun mezzo per imparare, si trattasse di libri o di conversazione. [...] Ero piuttosto alto, oltre la statura media. Senza essere, all'apparenza fisica, particolarmente atletico, o grande nelle dimensioni. Ero sorprendentemente vigoroso, ed attivo. I miei muscoli erano elastici ed ero conformato in modo tale da eccellere nei divertimenti giovanili. Mi piaceva leggere di fatti che fossero pieni di attività, e mi interessavano in particolare i racconti nei quali la capacità fisica o la forza erano mezzi che fornivano l'abilità a cui fare ricorso nelle fasi di difficoltà. Mi dedicai ad occupazioni pratiche $\mathrm{e}$ riservavo molto del mio tempo libero a tentativi di compiere qualche invenzione meccanica. ${ }^{17}$
\end{abstract}

Le due figure sembrano combaciare in un modo sorprendente, se messe l'una accanto all'altra. Si completano in un tutto ideale e perfetto. Si veda, ancora, la discussione che i due hanno un giorno, nella biblioteca di Falkland, sui meriti di Alessandro Magno. Caleb chiede a Falkland perché Alessandro sia stato nominato "il Grande", affermando che questi può essere certo famoso, ma, per certi crimini da lui commessi, non certo degno di essere ammirato dai posteri. Falkland, allora, difende appassionatamente la figura di Alessandro, chiedendo se cultura, sensibilità e gusto non sono qualità bastevoli per suscitare l'ammirazione generale, se si sia mai sentito di un altro uomo più valoroso, generoso e libero, appunto, di Alessandro, modello di onore, generosità e disinteresse, un uomo che per la coltivata liberalità della mente e l'ineguagliabile grandezza dei suoi progetti, deve richiamare solo l'attenzione e l'ammirazione di tutte le epoche. A queste considerazioni, Caleb risponde che la fama di Alessandro è stata

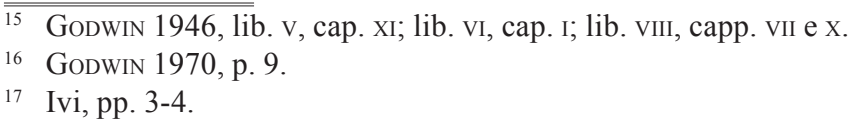


raggiunta a un prezzo troppo alto, cioè per mezzo di distruzioni, massacri, e crudeltà. Per lui, l'uomo è sicuramente una strana creatura se non ammira nessuno più vigorosamente di colui che ha disseminato distruzione e rovina sulla faccia della terra. ${ }^{18}$

E si noti che questa contrapposizione di giudizi - che, in effetti, è un reciproco completamento - non è un artificio retorico, per distinguere meglio quelli che sono personaggi centrali nel romanzo, dato che in base ai principi godwiniani ambedue le argomentazioni, quella culturale di Falkland e quella più "umanitaria" di Caleb, hanno diritto di cittadinanza e sono pienamente legittime.

È evidente che se, come ho affermato, Caleb e Falkland sono le parti separate di un'entità che le comprende, e solo la loro unione può formare l'uomo vero, una lettura soltanto psicanalitica del Caleb Williams, ${ }^{19}$ benché legittima, non è sufficiente: essa può avere qualche successo, in merito ai romanzi successivi di Godwin, ove al centro dell'azione non vi è la rappresentazione di una frattura dell'uomo o dell'umanità, ma il problematico rapporto di un personaggio, identificabile con Godwin e con il suo Io, con il mondo e gli altri uomini. Caleb, invece, non è la proiezione di Godwin, o lo è solo sino a un certo punto. L'Io narrante - Caleb - è qualcosa di complessivamente ben diverso dall' Io godwiniano, poiché Godwin si trova al di sopra della separazione che si incarna nei due personaggi, si pone proprio dove lo iato tra Falkland e Caleb si ricompone ed essi divengono quindi il "tutto", sconvolto dall'ordine sociale esistente, non più frammenti separati, ma totalità. Basta pensare alla Political Justice, con la sua fiducia nella possibilità di avvicinarsi alla società ideale, nella quale gli uomini saranno dediti alla semplicità dei costumi, alla sincerità e si dedicheranno a sviluppare le capacità intellettuali e l'amore per lo studio, per rendersi conto che Godwin è proprio al di là della scissione tra esteriorità e interiorità, tra l'essenza dell'uomo e le convenzioni ed istituzioni sociali vigenti al suo tempo.

L'alienazione e l'estraneità dei rapporti umani non sono una caratteristica del solo Falkland, che a causa del suo esagerato e viscerale senso dell'onore nega in fondo se stesso e vive solo per l'esteriorità, in difesa della sua appartenenza sociale, gelosamente e morbosamente attaccato a questa, e attento che nessuno possa gettare un'ombra sulla sua fama, che egli vuole trasmettere alla posterità immacolata, e non velata da un pur minimo sospetto. ${ }^{20}$

Anche Caleb è, tuttavia, alienato, a causa della conoscenza del segreto del padrone, per quella curiosità irrefrenabile egli vive per l'apparenza, per qualcosa che è al di fuori di lui, quindi non può più stabilire alcun rapporto con gli altri, ma è destinato a restare solo, senza amici, chiuso in se stesso, oggetto dei voleri di un'altra persona e non padrone di se stesso. È Falkland, rivolgendosi a Caleb, quando è costretto, dalla curiosità di questi, a confessare il delitto commesso, a sottolineare la perdita di indipendenza e di autonomia di Caleb, e in termini di perdita parallela della personalità, di "vendita": «per soddisfare un folle e curioso capriccio, hai venduto te stesso.

\footnotetext{
8 Ivi, pp. 110-111.

19 Cfr. ad esempio De Palacio 1980.

20 L'alienazione di Falkland è sottolineata da MıYoshi 1969, pp. 23-29.
} 
Continuerai così a essere al mio servizio, ma non potrai avere il mio affetto. Avrò cura del tuo futuro, ma ti odierò per sempre. Se mai dalle tue labbra dovesse sfuggire un'involontaria parola, se mai eccitassi la gelosia, oppure il mio sospetto, aspettati di pagare tutto questo con la morte, o peggio». ${ }^{21}$

Da questo momento, ha inizio l'impossibilità per Caleb di avere un rapporto normale col mondo che lo circonda, con le persone con le quali giunge in contatto, irrimediabilmente diviso, tra ciò che egli vorrebbe essere e fare e ciò che invece il suo padrone pretende che lui sia e faccia:

Ero un prigioniero: e quale prigioniero. Tutte le mie azioni osservate, e ogni mio gesto esaminato. Non potevo muovermi, né a destra, né a sinistra, senza che l'occhio del mio guardiano non fosse su di me. Mi sorvegliava; e la sua vicinanza faceva male al mio cuore. Per me non vi era più libertà, non vi era più allegria, spensieratezza, gioventù. ${ }^{22}$

Questa scissione dell'uomo in due entità distinte impedisce quindi che vi sia l'idillio, o una storia d'amore con l'amata Emily, mentre Caleb non potrà trovare la pace $\mathrm{o}$ rapporti umani, sinceri e naturali, nel paesaggio semplice e selvaggio, quasi roussoviano, del villaggio gallese dove egli si rifugia, in quel mondo sperduto dove vive la gente sincera, che sembra essere pienamente se stessa. Date le strutture sociali inique, che permettono e accentuano la frattura interiore dell'uomo, di tutti gli esseri umani, la felicità, come l'innocenza e la serenità, non possono essere raggiunte, e quei brevi momenti in cui esse sono assaporate sono sommersi dall'angoscia e dal terrore (ad esempio, la perdita di Laura).

Infine, Caleb e Falkland hanno in comune anche l'intelligenza viva, la curiosità, la voglia sana di conoscere, l'amore per la virtù. Del resto, questa comunanza è confermata dal reciproco rispetto, che mai viene a cessare, dalla devozione di Caleb verso Falkland, e dall'amore di questi nei confronti dello stesso Caleb: un patto implicito, ma costantemente affiorante, li lega, quello di non uccidersi, e di non farsi male, fisicamente e moralmente, di salvaguardare e curare l'altro, quanto più sia possibile. Oltre a ciò, i due sono accomunati dal segreto che li unisce e che mai confidano ad alcuno. Un segreto che è la causa del rimorso e della persecuzione di Falkland, ma che diviene contemporaneamente la causa della persecuzione e del rimorso di Caleb medesimo.

La morte del padrone-persecutore diviene così, necessariamente, la non-esistenza di Caleb, cioè un'altra morte, poiché i due non potevano vivere separati, lontani, senza l'altro "se stesso". Insomma, è il rimorso di avere perseguitato l'altro io, l'altra parte di un organico e unico "tutto", cercata e tuttavia non raggiunta, poiché la società con la sua ingiusta strutturazione - ancora, ritorna qui Rousseau - e i rapporti umani che si fondano su presupposti sbagliati, dividono nell'essenza più intima l'uomo e la sua esistenza, separando, violentemente, l'essere dal dover-essere, la verità dalla giustizia, l'esteriorità dalla interiorità, ciò che si è da ciò che si appare. E in questo modo si

\footnotetext{
$\overline{\overline{21} \text { GodwIN 1970, p. }} 136$.

22 Ivi, p. 143.
} 
costringe l'essere umano - l'umanità tutta - al dolore, alla persecuzione, al sospetto, al non-essere. ${ }^{23}$

Calogero Farinella a cura di Davide Arecco, Paolo Luca Bernardini, Laura Orsi

\section{RIFERIMENTI BIBLIOGRAFICI}

Adamo 2017 : P. Adamo, William Godwin e la società libera, Torino, Claudiana, 2017.

Argenton 1977 : A. Argenton, La concezione pedagogica di un classico dell'anarchismo: William Godwin, Bologna, Pàtron, 1977.

De Palacio 1980 : J. de Palacio, William Godwin et son monde interieur, Lille, Presses universitaires de Lille, 1980.

Di Piazza 1983 : E. Di Piazza, Caleb Williams. Un antagonismo imperfetto, Palermo, Flaccovio, 1983.

Dumas 1966 : D.G. Dumas, Things As They Are. The Original Ending of Caleb Williams, «Studies in English Literature»6, 3 (1966), pp. 575-597.

FLeISCHER 1951 : D. Fleischer, William Godwin. A Study in Liberalism, New York, Kelley, 1951.

Furbank 1955 : P.N. Furbank, Godwin's Novels, «Essays in Criticism» 5, 3 (1955), pp. 214-228.

GHIRARDELli 1993 : G. Ghirardelli, La visione politico-sociale di William Godwin attraverso l'opera The adventures of Caleb Williams or things as they are, tesi di dottorato, Milano, IULM, a.a. 1993 (tutor A. Beati).

Godwin 1912 : W. Godwin, Caleb Williams, Firenze, Bemporad, 1912.

GodwIn 1946 : W. Godwin, Enquiry Concerning Political Justice, Toronto, Toronto University Press, 1946.

Godwin 1970 : W. Godwin, Things As They Are, or the Adventures of Caleb Williams, Oxford, Oxford University Press, 1970.

Godwin 1975 : W. Godwin, Saint Leon, New York, AMS Press, 1975.

\footnotetext{
23 Sul pensiero politico godwiniano - nonché per un necessario e utile aggiornamento bibliografico, dato che Farinella compose questo suo contributo sul finire degli anni Ottanta del secolo scorso si vedano oggi ADAMO 2017. Inoltre, segnaliamo in questa sede la recente traduzione italiana anche dell'altra opera citata da Farinella: Godwin 2020. Cfr. altresì sui temi più strettamente filosofici del presente articolo, ARGENTON 1977 (conosciuto da Farinella ma non citato qui), e infine, per limitarci al solo panorama italiano (quello anglosassone presenta una letteratura vastissima negli ultimi quaranta anni), Ghirardelli 1993 [N.d.C.].
} 
Godwin 1976 : W. Godwin, Caleb Williams, Firenze, Valleccchi, 1976.

Godwin 1997 : W. Godwin, Caleb Williams, a cura di F. Ruggieri, Roma, Bagatto, 1997 (I ed. 1982).

Godwin 2018 : W. Godwin, Caleb Williams, o così va il mondo, a cura di R. Bicicchi, Rimini, Theoria, 2018.

Godwin 2020 : W. Godwin, St. Leon, l'alchimista, Roma, Haiku, 2020.

Kelly 1976 : G. Kelly, The English Jacobin Novel (1780-1805), Oxford, Oxford University Press, 1976.

Mryoshi 1969 : M. Miyoshi, The Divided Self, Toronto, Toronto University Press, 1969.

RotTA 1981 : S. Rotta, Maturazione e contraddizioni della cultura europea nell'Illuminismo, in Storia d'Italia e d'Europa. Comunità e popoli, a cura di M. Guidetti, v, Dall'ancien régime all'età napoleonica, Milano, Jaca Book, 1981, pp. 127-184. 